\title{
HPV monitoring in kidney transplanted patients
}

\author{
Franco M Buonaguro, MariaLina Tornesello, Luigi Buonaguro \\ From 13th International Conference on Malignancies in AIDS and Other Acquired Immunodeficiencies \\ (ICMAOI) \\ Bethesda, MD, USA. 7-8 November 2011
}

\section{Background}

Renal allograft recipients, as for HIV/AIDS patients, have a well-documented increased incidence of human papillomavirus (HPV)-related malignancies and preventive strategies should be specifically implemented. While in females the use of the Papanicolau test and HPV detection assay are used currently as a screening test for cervical cancer, no diagnostic procedures have been implemented to monitor HPV infection in males. The aim of this study was to test for HPV infection and to determine the spectrum of viral genotypes in urine samples of men with renal transplants.

\section{Material and methods}

The study included 103 patients who underwent kidney transplantation between 1999 and 2008. HPV sequences were detected by nested PCR, using the broad-spectrum consensus- primer pairs MY09/MY11 and the new MGP system, and characterized by nucleotide sequence analysis.

\section{Results}

Overall, 49 (47.5\%) samples were found positive for HPV sequences and the most common genotypes were HPV 16 (51.0\%) and HPV 54 (10.2\%) followed by HPV6, $53,56,58,66,11,12,20,45,62$, and 71 , in descending order of prevalence (Table 1). The majority of HPV 16 isolates were classified as European and only two as African-1 variant on the basis of nucleotide signature present within the MGP L1 region.

\section{Conclusion}

The high prevalence of HPV 16 among renal allograft recipients suggests that an HPV-16-based preventive or therapeutic vaccine may be effective for prevention or

\footnotetext{
* Correspondence: irccsvir@unina.it

Unit of Molecular Biology and Viral Oncogenesis \& AIDS Reference Center, Istituto Nazionale Tumori "Fondazione Giovanni Pascale", Naples, Italy
}

\begin{tabular}{ll}
$\begin{array}{l}\text { Table } 1 \text { Prevalence of HPV genotypes in renal transplant } \\
\text { patients }\end{array}$ \\
\hline HPV genotype $^{\text {a }}$ & HPV positive, $n(\%)$ \\
\hline HPV positive & $49(47.5)$ \\
HPV negative & $54(52.4)$ \\
Single infections & \\
16 & $25(51.0)$ \\
56 & $2(4.0)$ \\
58 & $1(2.0)$ \\
54 & $5(10.2)$ \\
6 & $2(4.0)$ \\
11 & $1(2.0)$ \\
12 & $1(2.0)$ \\
20 & $1(2.0)$ \\
53 & $1(2.0)$ \\
62 & $1(2.0)$ \\
66 & $1(2.0)$ \\
71 & $1(2.0)$ \\
Total single infections & $42(85.7)$ \\
Total multiple infections & $4(8.2)$ \\
Undetermined & $3(6.1)$ \\
\hline
\end{tabular}

${ }^{\mathrm{a}}$ Gray shadow indicates HPV genotypes defined by IARC working group as class I carcinogens for humans [Bouvard et al., Lancet Oncol 10:321-322, 2009]

treatment of HPV-related neoplasia in this group of immune compromised patients.

Published: 19 April 2012

Reference

1. Tornesello ML, Loquercio G, Tagliamonte M, Rossano F, Buonaguro L, Buonaguro FM: Human papillomavirus infection in urine samples from male renal transplant patients. J Med Virol 2010, 82:1179-1185.

doi:10.1186/1750-9378-7-S1-P15

Cite this article as: Buonaguro et al:: HPV monitoring in kidney transplanted patients. Infectious Agents and Cancer 2012 7(Suppl 1):P15. 